\title{
THE PICARD-LEFSCHETZ FORMULA AND A CONJECTURE OF KATO: THE CASE OF LEFSCHETZ FIBRATIONS
}

\author{
Caterina Consani and Minhyong Kim
}

\section{Introduction}

The rational cohomology groups of complex algebraic varieties possess Hodge structures which can be used to refine their usual topological nature. Besides being useful parameters for geometric classification, these structures reflect in essential ways the conjectural category of motives, and hence, are of great interest from the viewpoint of arithmetic. A key link here is provided by the Hodge conjecture, which says that for smooth projective varieties, a class of type $(n, n)$ in $H^{2 n}$ should come from an algebraic cycle. Stated slightly more abstractly, a copy of the trivial Hodge structure inside $H^{2 n}(X)(n)$ for a smooth projective variety $X$ should be generated by an algebraic cycle class on $X$. Obviously, for the Hodge structures coming from smooth projective varieties, $H^{2 n}(n)$ are the only ones that are of weight zero, and therefore, that can have trivial sub-structures.

On the other hand, there are many important mixed Hodge structures (MHS) ([2] [3]) that arise from algebraic geometry, including open or singular varieties, local cohomologies, and the cohomology of Milnor fibers. The presence of trivial substructures is likely to reflect subtler phenomena (than the Hodge conjecture!) in the mixed case, having to do (possibly in a complicated way) with trivial substructures of various pure sub-quotients.

The example we have in mind here is that of limit Hodge structures coming from degenerations $([5])$. That is, let $\Delta$ be the unit disk and

$$
X \rightarrow \Delta
$$

a flat projective family of varieties of fiber dimension $d$ whose only critical value is the origin and assume that the divisor $Y:=X_{0}$ has normal crossings. We also assume that the total space is non-singular of dimension $d+1$ and that the fundamental group of the punctured base $\pi_{1}\left(\Delta^{*}\right) \simeq \mathbf{Z}$ acts unipotently on the cohomology of a generic fiber. Let $\Delta^{*}=\Delta-\{0\}, X^{*}=X-X_{0}$ and $\bar{X}^{*}$ be the pull-back of $X^{*}$ to the universal cover $H$ (the upper-half plane) of $\Delta^{*}$ via the covering map $u \rightarrow \exp (2 \pi i u)$. Thus, $\bar{X}^{*}$ is homotopy equivalent to any smooth fiber $X_{t}$ of $X$. Then Steenbrink has put on $H^{n}\left(X_{t}\right) \simeq H^{n}\left(\bar{X}^{*}\right)$ a MHS via the theory of nearby cycles. That is, if we denote by $\bar{j}: \bar{X}^{*} \rightarrow X$ the natural map, $i: Y \hookrightarrow X$ the inclusion, and the complex of nearby cycles

$$
R \Psi:=i^{*} \bar{j}_{*} \mathbb{Q},
$$

Received July 9, 2002. 
one has isomorphisms

$$
H^{n}\left(X_{t}, \mathbb{Q}\right) \simeq H^{n}\left(\bar{X}^{*}, \mathbb{Q}\right) \simeq H^{n}(Y, R \Psi) \simeq H^{n}\left(Y,(R \Psi)_{u}\right)
$$

where $(R \Psi)_{u} \subset R \Psi$ denotes the maximal subcomplex on which the monodromy action is unipotent (one can make this precise with certain choices of complexes representing the objects in the derived category). The complex $(R \Psi)_{u}$ is shown to underlie a cohomological mixed Hodge complex (which depends on the choice of coordinate $u$ on $H$ ). An important consequence of the theory identifies the weight filtration for this MHS with the filtration given by the nilpotent operator $\log (T)$ where

$$
T: H^{n}\left(X_{t}\right) \rightarrow H^{n}\left(X_{t}\right)
$$

is the positive generator for the monodromy action of $\pi_{1}\left(\Delta^{*}\right)$ induced by the transformation $u \mapsto u+1$ of the upper half plane. Furthermore, $\log (T)$ shifts weights by -2 and Hodge types by $(-1,-1)$. More precisely, we have a map of (mixed) Hodge structures

$$
N:=-(1 / 2 \pi i) \log (T): H^{n}\left(X_{t}\right)(1) \rightarrow H^{n}\left(X_{t}\right)
$$

Using duality, we can therefore view $N$ as an element in

$$
\left(H^{n}\right)^{*} \otimes H^{n}\left(X_{t}\right)(-1) \simeq H^{2 d-n}\left(X_{t}\right) \otimes H^{n}\left(X_{t}\right)(d-1)
$$

generating a trivial substructure. Summing over all $n$ and using the Künneth formula, we get an element

$$
[N] \in H^{2 d}\left(X_{t} \times X_{t}\right)(d-1)
$$

generating a trivial substructure of the limit mixed Hodge structure on the cohomology of the product space $X_{t} \times X_{t}$. Now, weight considerations show that

$$
[N] \in\left[W_{2 d-2} H^{2 d}\left(X_{t} \times X_{t}\right)\right](d-1)
$$

thus giving rise to a trivial substructure of

$$
\left[\mathrm{Gr}_{2 d-2}^{W} H^{2 d}\left(X_{t} \times X_{t}\right)\right](d-1)
$$

The situation here is obviously different from the usual one for the Hodge conjecture in that we are not viewing the cohomology groups with the usual pure Hodge structure. Nonetheless, the construction of Steenbrink's spectral sequence provides a natural context for interpreting this situation. For this, let

$$
Z \rightarrow \Delta
$$

be a regular normal-crossing model for $X \times_{\Delta} X$, and let $S$ be its special fiber. It will be made up of smooth components that cross normally. Denote by

$$
S^{[i]}
$$

the disjoint union of all $i$-fold intersections of these components.

Then Steenbrink's spectral sequence expresses the pure graded pieces of $H^{2 d}\left(X_{t} \times X_{t}\right)$ as made up of the $E_{2}$ term of a spectral sequence, whose $E_{1}$ 
term consists of sums of cohomology groups of the $S^{[i]}$. In particular, the piece of interest

$$
\left[\mathrm{Gr}_{2 d-2}^{W} H^{2 d}\left(X_{t} \times X_{t}\right)\right]
$$

can be expressed as a subquotient of the cohomology of smooth projective varieties.

We can be more precise. For this, we note that $N$ viewed as a map

$$
H(X)(1) \rightarrow H(X)
$$

commutes with the monodromy action on the source and target, and hence, the class $[N]$ is invariant for the monodromy operator on the (twisted) cohomology of the product. Now, an important consequence of the theory (the invariant cycle theorem) expresses the monodromy invariant part of a limit MHS as the image of the MHS of the special fiber. That is, the natural map

$$
H^{2 d}(S) \rightarrow H^{2 d}\left(X_{t} \times X_{t}\right)
$$

surjects onto the invariant part. On the other hand, since the morphism of Hodge structures is strict, we get thereby a surjection

$$
\mathrm{Gr}_{2 d-2}^{W} H^{2 d}(S) \rightarrow \mathrm{Gr}_{2 d-2}^{W} H^{2 d}\left(X_{t} \times X_{t}\right)^{I}
$$

where we have denoted with the superscript $I$ the monodromy invariant part. We have

$\operatorname{Gr}_{2 d-2}^{W} H^{2 d}(S) \simeq \operatorname{Ker}\left[H^{2 d-2}\left(S^{[3]}\right) \rightarrow H^{2 d-2}\left(S^{[4]}\right)\right] / \operatorname{Im}\left[H^{2 d-2}\left(S^{[2]}\right) \rightarrow H^{2 d-2}\left(S^{[3]}\right)\right]$ where the maps are alternating sums of restriction maps (with signs determined by fixing an ordering of the components), weighted suitably by the multiplicities of the components. Therefore, there exists a trivial substructure of $H^{2 d-2}\left(S^{[3]}\right)(d-1)$ which maps to the subspace generated by $[N]$, and the Hodge conjecture would imply that this space can be represented by an algebraic cycle. The problem of identifying this algebraic cycle was posed by Kazuya Kato. Stated more precisely, he pointed out the following consequence of the Hodge conjecture:

Conjecture 1. There exists an algebraic cycle on $S^{[3]}$ whose cycle class lies in the kernel of the restriction map to $S^{[4]}$ and which maps to the class $[N]$ of the monodromy operator discussed above.

This conjecture was proved for the case of curves with double point degenerations and surfaces with triple point degenerations in [1] for a specific model $Z$. In fact, one of the results of the present paper is that:

Theorem 1.1. The conjecture is independent of the model $Z$ of $X \times_{\Delta} X$. That is, if the conjecture is verified for one normal-crossing model $Z$ then the conjecture holds for any other normal-crossing model $Z^{\prime}$.

Furthermore, we will prove the conjecture in the very simple case of semistable fibrations arising from isolated quadratic singularities. That is, assume that $V^{\prime} \rightarrow \Delta$ is a projective flat map where the singularities of the map are all in the special fiber $W^{\prime}$ and are isolated non-degenerate quadratic. Let $V$ be 
obtained by blowing up the singular points of $W^{\prime}$. Finally, let $X$ be obtained by normalizing the base change of $V$ via the squaring map $\Delta \rightarrow \Delta$. Thus, $X$ is semi-stable, and we will have a monodromy class $[N] \in H^{2 d}\left(X_{t} \times X_{t}\right)(d-1)$.

Theorem 1.2. Kato's conjecture is true in this setting. That is, there is an algebraic cycle class in $H^{2 d-2}\left(S^{[3]}\right)(d-1)$ mapping via the Steenbrink spectral sequence to the class $[N]$.

The proof is a simple application of the Picard-Lefschetz formula and can easily be guessed at by experts. On the other hand, since the method suggests an approach to the general problem with possibly considerable technical advantages over the approach of [1], we considered it worth writing down explicitly.

This problem has an analogue in the case of mixed-characteristic degenerations that we do not treat here. But we note that the monodromy operator is the subject of one of the most important open problems of arithmetic geometry, namely, the weight-monodromy conjecture. It is our hope (perhaps futile) that the algebraicity of Kato's conjecture may eventually contribute to its resolution.

\section{Proofs}

Proof of Theorem 1. By the weak factorization theorem [6], any two normal crossing models (that is, regular models having normal crossings in the special fiber) can be connected by a sequence of diagrams

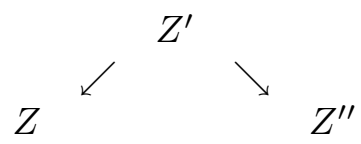

where each arrow is either the identity or the blow-up along a non-singular center in the special fiber. Also, we can assume all the models occuring in the diagram are normal crossing and that the center of each blow-up meets the components of the special fiber normally. Thus, by iterating along the diagram, we need only show that the algebraicity of the monodromy class $[N]$ for the two models $Z$ and $Z^{\prime}$ are equivalent. Denote the map by $f: Z^{\prime} \rightarrow Z$ and let $E$ be the exceptional divisor.

Let us choose some complex computing the cohomology of the Steenbrink complexes for the two models, say that constructed using $C^{\infty}$ differential forms. That is, we let $A$ and $A^{\prime}$ be the global $C^{\infty}$ versions of the Steenbrink complexes for $Z$ and $Z^{\prime}$, where the sheaf of $\log$ differential forms is replaced by global $C^{\infty}$ differential forms with log poles. We clearly have a map $f^{*}: A \rightarrow A^{\prime}$ which preserves the weight and Hodge filtrations. Thus, we have a map of the Steenbrink spectral sequences associated to the two models.

$$
f^{*}: E_{1}^{-r, q+r}(Z) \rightarrow E_{1}^{-r, q+r}\left(Z^{\prime}\right), \quad f_{!}: E_{1}^{-r, q+r}\left(Z^{\prime}\right) \rightarrow E_{1}^{-r, q+r}(Z) .
$$

By the description of the associated graded pieces via residues it follows that the pull-back map at the $E_{1}$ level is given by usual pullback in cohomology for 
the components corresponding to various components of strata. That is, at the rational level, these morphisms are described by a direct sum of maps as

$$
f^{*}: H^{q-r-2 k}\left(S^{[2 k+r+1]}, \mathbb{Q}(-r-k)\right) \rightarrow H^{q-r-2 k}\left(S^{[2 k+r+1]}, \mathbb{Q}(-r-k)\right),
$$

where $k$ is an integer satisfying $k \geq \max (0,-r)$.

Since both spectral sequences are associated to a mixed Hodge complex converging to the cohomology of the generic fiber, we see that $f^{*}$ is a quasiisomorphism when we regard $E_{1}(Z)$ and $E_{1}\left(Z^{\prime}\right)$ as complexes. We can in fact construct a splitting $f_{!}: E_{1}\left(Z^{\prime}\right) \rightarrow E_{1}(Z)$ as follows. The components of $\left(S^{\prime}\right)^{[i]}$ are of the form

$$
S_{a_{1}}^{\prime} \cap \cdots \cap S_{a_{i}}^{\prime}=\left(S_{a_{1}} \cap \cdots \cap S_{a_{i}}\right)^{\prime}
$$

and

$$
S_{a_{1}}^{\prime} \cap \cdots \cap S_{a_{i-1}}^{\prime} \cap E=\left(S_{a_{1}} \cap \cdots \cap S_{a_{i-1}}\right)^{\prime} \cap E
$$

where $S_{k}^{\prime}$ is the strict transform of the component $S_{k}$ of $S$. Here, we have chosen the ordering of the components of $S^{\prime}$ in such a way that it is compatible with the ordering of the $S_{k}$ 's and so that $E$ is the last element. Then we define $f_{\text {! }}$ on

$$
H^{n}\left(S_{a_{1}}^{\prime} \cap \cdots \cap S_{a_{i}}^{\prime}\right)
$$

as the usual push-forward to

$$
H^{n}\left(S_{a_{1}} \cap \cdots \cap S_{a_{i}}\right)
$$

and on

$$
H^{n}\left(S_{a_{1}}^{\prime} \cap \cdots \cap S_{a_{i-1}}^{\prime} \cap E\right)
$$

as zero.

Let us check that $f_{!}$commutes with the differentials on $E_{1}\left(Z^{\prime}\right)$ and $E_{1}(Z)$. On both complexes the differential $d$ has two components $d_{1}$ and $d_{2}$, the first consisting of restriction maps and the other of Gysin maps. (There is a slight complication coming from the weighting for the multiplicities. But these will be the compatible on the two complexes because the center of the blow-up meets the divisor $S$ normally, so we will ignore them.)

Assume we start from a class

$$
c \in H^{n}\left(S_{a_{1}}^{\prime} \cap \cdots \cap S_{a_{i}}^{\prime}\right)
$$

The various restriction maps will have some components in groups of the form

$$
H^{n}\left(S_{a_{1}}^{\prime} \cap \cdots \cap S_{a_{i}}^{\prime} \cap S_{a_{i+1}}^{\prime}\right)
$$

for which we have a commutative diagram

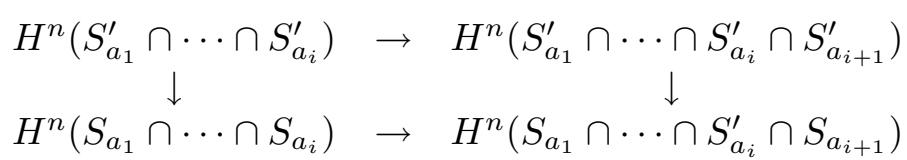

To see that this diagram is commutative, describe the map

$$
f_{*}: H^{n}\left(S_{a_{1}}^{\prime} \cap \cdots \cap S_{a_{i}}^{\prime}\right) \rightarrow H^{n}\left(S_{a_{1}} \cap \cdots \cap S_{a_{i}}\right)
$$


as follows: If $x \in H^{n}\left(S_{a_{1}}^{\prime} \cap \cdots \cap S_{a_{i}}^{\prime}\right)$, then $x=f^{*}(y)+z$ for a unique $y \in$ $H^{n}\left(S_{a_{1}} \cap \cdots \cap S_{a_{i}}\right)$ and $z \in \operatorname{Ker}\left(f_{*}\right)$. Then $f_{*}(x)=y$. We have an analogous description of the map

$$
H^{n}\left(S_{a_{1}}^{\prime} \cap \cdots \cap S_{a_{i}}^{\prime} \cap S_{a_{i+1}}^{\prime}\right) \rightarrow H^{n}\left(S_{a_{1}} \cap \cdots \cap S_{a_{i}} \cap S_{a_{i+1}}\right)
$$

So one sees easily that it suffices to check that the restriction of $z$ to $H^{n}\left(S_{a_{1}}^{\prime} \cap \cdots \cap\right.$ $\left.S_{a_{i}}^{\prime} \cap S_{a_{i+1}}^{\prime}\right)$ also lies in the kernel of $f_{*}$. But this follows from the commutative diagram

$$
\begin{array}{ccc}
H^{n-2}\left(S_{a_{1}}^{\prime} \cap \cdots \cap S_{a_{i}}^{\prime} \cap E\right) & \rightarrow & H^{n}\left(S_{a_{1}}^{\prime} \cap \cdots \cap S_{a_{i}}^{\prime}\right) \\
H^{n-2}\left(S_{a_{1}}^{\prime} \cap \cdots \cap S_{a_{i}}^{\prime} \cap S_{a_{i+1}}^{\prime} \cap E\right) & \rightarrow & H^{n}\left(S_{a_{1}}^{\prime} \cap \cdots \cap S_{a_{i+1}}^{\prime}\right)
\end{array}
$$

which comes from the functoriality of the localization exact sequence, and the fact that the images of these horizontal maps are exactly the kernels of the push-forward maps. On the other hand, the restriction to components of the form

$$
H^{n}\left(S_{a_{1}}^{\prime} \cap \cdots \cap S_{a_{i}}^{\prime} \cap E\right)
$$

composes to zero under $f_{!}$. This shows that $f_{!}\left(d_{1}(c)\right)=d_{1} f_{!}(c)$. The other compatibility, $f_{!}\left(d_{2}(c)\right)=d_{2} f_{!}(c)$ just follows from the functoriality of pushforwards.

Now start from

$$
c \in H^{n}\left(S_{a_{1}}^{\prime} \cap \cdots \cap S_{a_{i-1}}^{\prime} \cap E\right) .
$$

Then both $f_{!} d_{1}$ and $d_{1} f_{!}$are zero. Also, $d_{2} f_{!}(c)=0$ by definition. So to conclude, we need only check that $f_{!} d_{2}(c)$ is zero. This is clear for the components of $d_{2}$ corresponding to Gysin maps of the form

$$
H^{n}\left(S_{a_{1}}^{\prime} \cap \cdots \cap S_{a_{i}}^{\prime} \cap E\right) \rightarrow H^{n+2}\left(S_{b_{1}}^{\prime} \cap \cdots \cap S_{b_{i-1}}^{\prime} \cap E\right)
$$

For the map

$$
H^{n}\left(S_{a_{1}}^{\prime} \cap \cdots \cap S_{a_{i}}^{\prime} \cap E\right) \rightarrow H^{n+2}\left(S_{a_{1}}^{\prime} \cap \cdots \cap S_{a_{i}}^{\prime}\right)
$$

note that

$$
H^{n+2}\left(S_{a_{1}}^{\prime} \cap \cdots \cap S_{a_{i}}^{\prime}\right) \simeq H^{n+2}\left(S_{a_{1}}^{\prime} \cap \cdots \cap S_{a_{i}}^{\prime}\right) \oplus H^{n}\left(S_{a_{1}}^{\prime} \cap \cdots \cap S_{a_{i}}^{\prime} \cap E\right)
$$

by the formula for the cohomology of a blow-up and that $f_{!}$is just projection onto the first component while the second component is the image of the Gysin map. Thus, $f_{!} d_{2}(c)=0$ and we are done.

We can write now

$$
E_{1}\left(Z^{\prime}\right) \simeq E_{1}(Z) \oplus K
$$

as complexes where $K$ is the kernel of $f$ ! and is acyclic. In particular, we see that both $f^{*}$ and $f_{\text {! }}$ induce maps between

$$
\operatorname{Ker}\left(d: E_{1}(Z) \rightarrow E_{1}(Z)\right)
$$

and

$$
\operatorname{Ker}\left(d: E_{1}\left(Z^{\prime}\right) \rightarrow E_{1}\left(Z^{\prime}\right)\right)
$$


which are compatible with maps from either to

$$
\mathrm{Gr}^{W}\left(H^{*}\left(X_{t} \times X_{t}\right)\right) \text {. }
$$

Finally, noting that both $f^{*}$ and $f$ ! take algebraic classes to algebraic classes allows us to conclude the proof.

Proof of Theorem 2. We start by reviewing the relevant portions of the PicardLefschetz formalism ([4] XV).

To restate our assumptions, let $V^{\prime} \rightarrow \Delta$ be a proper flat map, smooth over $\Delta^{*}$. We now assume that $V^{\prime}$ has odd fiber dimension $d$ and that the singularities of the special fiber are all quadratic and isolated, coming from a local expression $\underline{x} \rightarrow Q(\underline{x})$ for our map $V^{\prime} \rightarrow \Delta$, where $Q$ is a non-degenerate quadratic form. We note at this point that the case of even fiber dimensions is trivial with respect to our problem since the monodromy operator is then zero. Let $V \rightarrow V^{\prime}$ be the blow-up of $V^{\prime}$ along the singularities of the special fiber.Denote by $W^{\prime}$ and $W$ the special fibers of $V^{\prime}$ and $V$ respectively. We see that $W$ is normal crossing with $s+m$ components, where $m$ is the number of components of $W^{\prime}$ and $s$ is the number of singularities in the special fiber $W^{\prime}$. Note that $m=1$ unless $d=1$ with our assumptions. For the rest of this paper, we will therefore assume that $d>1$ and hence $m=1$, since the arguments for $d=1$ simply involve omitting some steps in an obvious manner, and another proof is given in [1] in any case.

Let $X \rightarrow \Delta$ be obtained from $V$ by changing base with respect to the map $\Delta \rightarrow \Delta, \quad t \mapsto t^{2}$ and normalizing. Thus, $X$ is a smooth manifold and the special fiber $Y$ is reduced normal crossing. The set of its components are in bijection with the components of $W$, and we label them $\left\{Y_{i}\right\},\left\{W_{i}\right\}$, for some label set to be determined later, so that the map $X \rightarrow V$ maps $Y_{i}$ to $W_{i}$. Let $T^{\prime}$ be the monodromy operator on the cohomology of $V_{t}^{\prime}$, any smooth fiber of $V^{\prime}$. Thus, $T^{\prime}$ acts non-trivially only on $H^{d}\left(V_{t}^{\prime}\right)$. Then the Picard-Lefschetz formula says

$$
T^{\prime}(x)=x-\Sigma_{p}\left(\delta_{p}, x\right) \delta_{p}
$$

where $p$ runs over the singularities of $V_{0}^{\prime}$ and $\delta_{p} \in H^{d}\left(V_{t}^{\prime}\right)$ is the vanishing cycle associated to $p$. $\delta_{p}$ is determined (up to sign) as follows: we have the composed map

$$
H_{p}^{d}\left(W^{\prime}\right) \rightarrow H^{d}\left(W^{\prime}\right) \rightarrow H^{d}\left(V_{t}^{\prime}\right)
$$

and $H_{p}^{d}\left(W^{\prime}\right)$ is free of rank one. Take $\delta_{p}$ to be the image of any generator of $H_{p}^{d}\left(W^{\prime}\right)$. The formula is clearly independent of the choice of generator. Now, if $T$ denotes the monodromy operator for $X$ acting on $H^{d}\left(X_{t}\right)=H^{d}\left(V_{t^{2}}^{\prime}\right)$, then we have $T=\left(T^{\prime}\right)^{2}$ and $T^{\prime}$ acts trivially on the vanishing cycles, so we get

$$
T(x)=x-\Sigma_{p} 2\left(\delta_{p}, x\right) \delta_{p}
$$

Therefore, the log of the monodromy is given by

$$
\log T(x)=T(x)-x=-\Sigma_{p} 2\left(\delta_{p}, x\right) \delta_{p}
$$

since $(T-1)^{2}=0$, and the cohomology class $[N]$ in

$$
H^{2 d}\left(X_{t} \times X_{t}\right)(d-1)
$$


defined by $N=-(1 / 2 \pi i) \log T$ is the class

$$
(2 \pi i)^{d-1} 2 \Sigma_{p} \delta_{p} \otimes \delta_{p} \in H^{d}\left(X_{t}\right) \otimes H^{d}\left(X_{t}\right)(d-1) \subset H^{2 d}\left(X_{t} \times X_{t}\right)(d-1)
$$

We will show, in fact, that every class $(2 \pi i)^{d-1} \delta_{p} \otimes \delta_{p}$ is 'algebraic' in the sense described in the introduction. For this, we take $Z$ to be the blow-up of $X \times_{\Delta} X$ along its singular locus, which we will describe more precisely below. We have a commutative diagram

$$
\begin{array}{cccc}
X_{t} \times X_{t} & \hookrightarrow & Z \\
\| & & \downarrow \\
X_{t} \times X_{t} & \hookrightarrow & X \times_{\Delta} X
\end{array}
$$

inducing a commutative diagram of maps in cohomology

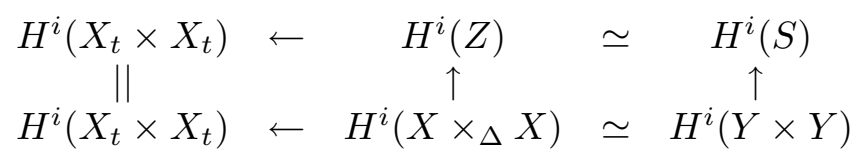

and we need to show that the class $[N]$ in $H^{2 d}\left(X_{t} \times X_{t}\right)(d-1)$ comes from an algebraic class in $H^{2 d-2}\left(S^{[3]}\right)(d-1)$. To get this, we need to analyze a bit the original Lefschetz fibration $V^{\prime}$. The class $\delta_{p} \in H^{d}\left(V_{t}^{\prime}\right)$ comes from a class $\epsilon_{p} \in H^{d}\left(W^{\prime}\right)$ which in turn comes from the (rank 1) local cohomology $H_{p}^{d}\left(W^{\prime}\right)$ via the map $H_{p}^{d}\left(W^{\prime}\right) \rightarrow H^{d}\left(W^{\prime}\right)$. On the blowup $V$, the special fiber $W$ contains a component $W_{p}$ mapping to the point $p$ for each $p$ and there is a component $W_{0}$ given by the strict transform of $W^{\prime}$. Also, $W_{0 p}:=W_{0} \cap W_{p}$ is the exceptional divisor of the (blow-up) map $W_{0} \rightarrow W^{\prime}$. There is a surjection $H^{d-1}\left(W_{0 p}\right) \rightarrow H_{p}^{d}\left(W^{\prime}\right)$ defined as follows: Let $U_{p}$ be a contractible neighborhood of $p$ in $W^{\prime}$ and $N_{p}$ its inverse image in $W_{0}$. Then $N_{p}$ is homotopy equivalent to $W_{0 p}$ while $N_{p}-W_{0 p} \simeq U_{p}-p$. So we have maps

$H^{d-1}\left(W_{0 p}\right) \simeq H^{d-1}\left(N_{p}\right) \rightarrow H^{d-1}\left(N_{p}-W_{0 p}\right) \simeq H^{d-1}\left(U_{p}-p\right) \rightarrow H_{p}^{d}\left(U_{p}\right)=H_{p}^{d}\left(W^{\prime}\right)$

But $H^{d-1}\left(W_{0 p}\right)$ is the middle degree cohomology of the smooth quadric $W_{0 p}$ and therefore ([4] XII.3.3) is generated by a power of the hyperplane class and one other class $\alpha_{p}^{\prime}$ (corresponding to the maximal isotropic subspaces for the quadratic form defining $\left.W_{0 p}\right)$ representing the primitive quotient. We can take $e_{p}^{\prime} \in H^{d}\left(W^{\prime}\right)$ to be the image of $\alpha_{p}^{\prime}$. Now, going back up to $X$, we therefore get classes $\alpha_{p} \in H^{d-1}\left(Y_{0 p}\right)$ where $Y_{0 p}$ is the intersection $Y_{0} \cap Y_{p}$ mapping to the pullback $e_{p}$ of $e_{p}^{\prime}$ to $H^{d}(Y)$. By construction, $2 \Sigma_{p} e_{p} \otimes e_{p} \in H^{2 d}(Y \times Y)$ maps to $[N]$ via the map to the generic fiber of $X \times_{\Delta} X$. Denote by $c_{p}$ the class in $H^{2 d}(S)$ obtained by pulling back $e_{p} \otimes e_{p}$ which, therfore, is the same as

$$
p_{1}^{*}\left(e_{p}\right) \cup p_{2}^{*}\left(e_{p}\right) \in H^{2 d}(S)
$$

where $p_{i}$ are the composition of the blow-up map $S \rightarrow Y \times Y$ with the projection maps to the two factors. Thus, $(2 \pi i)^{d-1} 2 \Sigma_{p} c_{p}$ is now the class in the cohomology of $S$ which maps to the class $[N]$. We wish to show that $c_{p}$ comes from an algebraic class in $H^{2 d-2}\left(S^{[3]}\right)(d-1)$. For this, we need to analyze a bit the geometry of the blow-up $Z \rightarrow X \times_{\Delta} X$. For each index $i$, either 0 or $p$, we have 
a component $Y_{i}$ of $Y$, giving us components $Y_{i} \times Y_{j}$ of $Y \times Y$. We also have the singular locus

$$
Y_{i j} \times Y_{l k}
$$

(using the obvious notation of double indices for the double intersections) of $X \times_{\Delta} X$ which we need to blow up to get $Z$. We will find the appropriate classes in the inverse image of $Y_{0 p} \times Y_{0 p}$.

We need only deal with each $p$ separately, so we will now fix a $p$ of interest and index the components of $S$ as follows: $S_{0}$ refers to the exceptional divisor mapping to $Y_{0 p} \times Y_{0 p} . S_{1}, S_{2}, S_{3}, S_{4}$ are then the strict transforms of $Y_{0} \times Y_{0}, Y_{0} \times$ $Y_{p}, Y_{p} \times Y_{0}$, and $Y_{p} \times Y_{p}$ respectively. If we note that the center of the blow-up is a Cartier divisor on $\left(Y_{0} \times Y_{0}\right) \cap\left(Y_{0} \times Y_{p}\right)$, we get that $S_{012}=S_{0} \cap S_{1} \cap S_{2}$ maps isomorphically to the center $Y_{0 p} \times Y_{0 p}$. Similarly, $S_{013}, S_{024}$, and $S_{034}$ map isomorphically to $Y_{0 p} \times Y_{0 p}$. Furthermore, these are the only non-empty triple intersections. Now we claim that there is an algebraic class in

$$
H^{2 d-2}\left(S_{013}\right)(d-1)
$$

which maps to $(2 \pi i)^{d-1} c_{p}$. To see this, we need some explicit computation with cocycles which describe the various maps involved.

First, let us describe the map

$$
f_{1}: H^{d-1}\left(W_{0 p}\right) \rightarrow H^{d}(W)
$$

obtained as the composite

$$
H^{d-1}\left(W_{0 p}\right) \rightarrow H^{d}\left(W^{\prime}\right) \rightarrow H^{d}(W)
$$

We claim it is the same as the map

$$
f_{2}: H^{d-1}\left(W_{0 p}\right) \rightarrow H^{d}(W)
$$

obtained from the spectral sequence for the hypercover

$$
\coprod W_{i} \rightarrow W
$$

To see this, we recall how to compute $f_{1}$ at the level of cocyles. Denote by $f$ the map $W \rightarrow W^{\prime}$ as well as its restriction to various subsets. Given a class $[x] \in H^{d-1}\left(W_{0 p}\right)$, let $x$ be a cocycle representing it. We can lift it to a cocycle $y$ on $N_{p}$ and then restrict it to $y_{0}$ on $N_{p}-W_{0 p}$ which can then be expressed as $f^{*}\left(y_{0}^{\prime}\right)$ for some cocycle $y_{0}^{\prime}$ on $U_{p}-p$. To get the image in $H_{p}^{d}\left(U_{p}\right)$, we just consider the relative cocycle $\left(0, y_{0}^{\prime}\right)$ in the cone complex computing local cohomology. Now we need to find a cocycle representing the class in $H_{p}^{d}\left(W^{\prime}\right) \simeq H_{p}^{d}\left(U_{p}\right)$. To do this, we solve $\left[\left(0, y_{0}^{\prime}\right)\right]=[(z, w)] \mid\left(U_{p}, U_{p}-p\right)$ where $z$ is a $d$-cocycle on $W^{\prime}$ and $w$ is a $d-1$ cochain on $W^{\prime}-p$ such that $z \mid\left(W^{\prime}-p\right)=d w$ and the equality of classes means that there is a pair $(a, b)$ consisting of a $d-1$ cochain $a$ on $U_{p}$ and a $d-2$ cochain $b$ on $U_{p}-p$ solving

$$
z\left|U_{p}=d a, \quad w\right|\left(U_{p}-p\right)=y_{0}^{\prime}+a\left|\left(U_{p}-p\right)-d b\right|\left(U_{p}-p\right)
$$

Then $f_{1}([x])$ is the class of $f^{*}(z)$ on $W$. 
In the above, notice that we can take $b=0$. This is done as follows: Consider the covering $W^{\prime}=\left(W^{\prime}-p\right) \cup\left(U_{p}\right)$. Then the complex underlying the MayerVietoris sequence for this covering allows us to write $y_{0}^{\prime}=w-a$ for a cochain $w$ that comes from $W^{\prime}-p$ and $a$ that comes from $U_{p}$. Since, $y_{0}^{\prime}$ is a cocycle, we get $d w=d a$ on $U_{p}-p$, giving us a class $z$ on $W^{\prime}$ satisfying $z=d w$ on $W^{\prime}-p$ and $z=d a$ on $U_{p}$. Then $(z, w)$ is a cocycle for relative cohomology satisfying the constraints above for $b=0$.

Pulling back to $W_{0}$ using $f$, we get an equality

$$
f^{*}(w)\left|\left(N_{p}-W_{0 p}\right)=\left(y+f^{*}(a)\right)\right|\left(N_{p}-W_{0 p}\right)
$$

the point being that on $W_{0}, f^{*}\left(y_{0}^{\prime}\right)=y_{0}$ extends to the class $y$ on $N_{p}$. So we get a class $v$ on $W_{0}$, defined to be $f^{*}(w)$ on $W_{0}-W_{0 p}$ and $y+f^{*}(a)$ on $N_{p}$, with the property that $v \mid W_{0 p}=x$. Then $f_{1}([x])$ is represented by the cocycle $f^{*}(z)$ on $W$, which is characterized by the property of restricting to $d v$ on $W_{0}$ and 0 on $W_{p}$.

On the other hand, we compute $f_{2}$ as follows. Let $w_{0 p}$ be a $d-1$ cocycle on $W_{0 p}$. Find cochains $w_{0}$ and $w_{p}$ on $W_{0}$ and $W_{p}$ such that $w_{0 p}=w_{0}-w_{p}$ on $W_{0 p}$. So we have a cochain $\left(w_{0}, w_{p}\right)$ in the direct sum of the complexes computing the cohomology of $W_{0}$ and $W_{1}$. Then compute its boundary to get $\left(d w_{0}, d w_{1}\right)$ which comes from a $d$ cocycle $w$ on $W$, that is, $w \mid W_{0}=d w_{0}$ and $w \mid W_{p}=d w_{p}$. Then $f_{2}\left(\left[w_{0 p}\right]\right)=[w]$. To compare the two maps, start with the cocycle $x$ on $W_{0 p}$. Above, we constructed a cochain $v$ on $W_{0}$ such that $v \mid W_{0 p}=x$. So we get the element $(v, 0) \in C^{d-1}\left(W_{0}\right) \oplus C^{d-1}\left(W_{p}\right)$ such that $v-0=x$ on $W_{0 p}$. Take the differential to get $(d v, 0)$. As noted above, $f_{1}([x])$ is represented by the cocycle $f^{*}(z)$ which is a class on $W$ that restricts to zero on $W_{p}$ and $d v$ on $W_{0}$. This shows that $f_{1}([x])=f_{2}([x])$.

Next, consider the map

$$
H^{d-1}\left(Y_{0 p}\right) \otimes H^{d-1}\left(Y_{0 p}\right) \rightarrow H^{2 d}(Y \times Y) \rightarrow H^{2 d}(S)
$$

In order to analyze the class $c_{p}$, we need to compare this with the map

$$
H^{2 d-2}\left(S_{012}\right) \oplus H^{2 d-2}\left(S_{013}\right) \oplus H^{2 d-2}\left(S_{024}\right) \oplus H^{2 d-2}\left(S_{034}\right) \rightarrow H^{2 d}(S)
$$

coming from the spectral sequence of the hypercover

$$
\coprod S_{i} \rightarrow S
$$

(Here, since we're speaking of a full hypercover of $S$ although we will be interested only in the part coming from $S_{0}, S_{1}, S_{2}, S_{3}, S_{4}$, we assume that we have extended the labelling to all of the components of $S$ in some manner.) Take the class $c_{p}=p_{1}^{*}\left(e_{p}\right) \otimes p_{2}^{*}\left(e_{p}\right) \in H^{2 d}(S)$ and restrict to the components $S_{0}, S_{1}, S_{2}, S_{3}, S_{4}$. For convenience of notation, we will suppress the pull-back maps and write, for example, $c_{p}=e_{p} e_{p}$. Recall that $S_{0}$ is the exceptional divisor of the blow-up $Z \rightarrow X \otimes_{\Delta} X$ while $S_{1}, S_{2}, S_{3}, S_{4}$ are the strict transforms of $Y_{0} \times Y_{0}, Y_{0} \times Y_{p}, Y_{p} \times Y_{0}$, and $Y_{p} \times Y_{p}$ respectively. The center of the blow-up is $Y_{0 p} \times Y_{0 p}$. Furthermore, we have represented $e_{p}$ as a cocycle characterized by $e_{p}\left|Y_{0}=d v, e_{p}\right| Y_{p}=0$, where $v$ is a cochain on $Y_{0}$ satisfying $v \mid Y_{0 p}=x$. Now, 
the restriction of $c_{p}=e_{p} e_{p}$ to $S_{0}$ is the same as taking $p_{1}^{*}\left(e_{p}\right) p_{2}^{*}\left(e_{p}\right)$ on $Y \times Y$, restricting to $Y_{0 p} \times Y_{0 p}$ and pulling back to the exceptional divisor. So $c_{p} \mid S_{0}=0$. Arguing this way with the various restrictions and using the fact that $d v \mid Y_{p}=0$, we see that the only component that survives is $c_{p} \mid S_{1}=d v d v=d(v d v)$. That is, we have the class

$$
(0, v d v, 0,0,0) \in C^{2 d-1}\left(S_{0}\right) \oplus C^{2 d-1}\left(S_{1}\right) \oplus C^{2 d-1}\left(S_{2}\right) \oplus C^{2 d-1}\left(S_{3}\right) \oplus C^{2 d-1}\left(S_{4}\right)
$$

whose differential is the restriction of $c_{p}$ to the components. Now,taking the Cech differential, by the same argument as above using $d v \mid Y_{p}=0$, we get that the only component among the double intersections that survives is $-v d v$ on $S_{13}$. This is the differential of the $2 d-2$ cochain $-v v$ on $S_{13}$. The Cech differential of this element has non-zero component only on $S_{013}$ which is equal to $-\pi^{*}\left(p_{1}^{*}\left(\alpha_{p}\right) p_{2}^{*}\left(\alpha_{p}\right)\right)$ for the isomorphism $\pi: S_{013} \rightarrow Y_{0 p} \times Y_{0 p}$. We get that $(2 \pi i)^{d-1}$ times this class is clearly algebraic, since $(2 \pi i)^{(d-1) / 2} a_{p}$ is algebraic. Then $(2 \pi i)^{d-1} c_{p}$, which is the image of

$$
(2 \pi i)^{d-1} \pi^{*}\left(p_{1}^{*}\left(\alpha_{p}\right) p_{2}^{*}\left(\alpha_{p}\right)\right)
$$

under the map

$$
H^{2 d-2}\left(S_{013}\right)(d-1) \rightarrow H^{2 d}(S)(d-1)
$$

is algebraic, and hence, so is $[N]$. We remark that the sign may differ from that given above depending on a convention for ordering various components.

\section{Acknowledgements}

The first author was supported in part by NSERC grant 72016789. The second author was supported in part by the NSF, and is grateful to the Korea Institute for Advanced Study and the University of Toronto for their hospitality. Both authors wish to thank the MPIM where part of this work was completed.

\section{References}

[1] C. Consani, The local monodromy as a generalized algebraic correspondence, Doc. Math. 4 (1999), 65-108 (electronic).

[2] P. Deligne, Théorie de Hodge. II, Inst. Hautes Études Sci. Publ. Math. No. 40 (1971), $5-57$.

[3] Théorie de Hodge. III, Inst. Hautes Études Sci. Publ. Math. No. 44 (1974), 5-77.

[4] P. Deligne, N. Katz, Groupes de monodromie en géométrie algébrique. II, Séminaire de Géométrie Algébrique du Bois-Marie 1967-1969 (SGA 7 II). Lecture Notes in Mathematics, Vol. 340. Springer-Verlag, Berlin-New York, 1973.

[5] J. H. Steenbrink, Limits of Hodge structures, Invent. Math. 31 (1975/76), 229-257.

[6] J. Włodarczyk, Toroidal varieties and the weak factorization theorem, preprint; math. AG/9904076

Department of Mathematics, University of Toronto, Toronto, Ontario, Canada M5S 3G3.

E-mail address: kc@math.toronto.edu

Department of Mathematics, University of Arizona, Tucson, AZ 85721, U.S.A.

E-mail address: kim@math.arizona.edu 